\title{
RUNX3 inhibits the metastasis and angiogenesis of colorectal cancer
}

\author{
BO RAM KIM ${ }^{1 *}$, MYOUNG HEE KANG $^{4 *}$, JUNG LIM KIM $^{2}$, YOO JIN NA ${ }^{1}$, SEONG HYE PARK ${ }^{1}$, \\ SUN IL LEE ${ }^{3}$, SANGHEE KANG ${ }^{3}$, SUNG YUP JOUNG ${ }^{3}$, SUK-YOUNG LEE ${ }^{2}$, \\ DAE-HEE LEE ${ }^{1,2}$, BYUNG WOOK MIN ${ }^{3}$ and SANG CHEUL OH ${ }^{1,2}$ \\ ${ }^{1}$ Graduate School of Medicine, Korea University College of Medicine; ${ }^{2}$ Division of Oncology/Hematology, \\ Department of Internal Medicine, Korea University College of Medicine; ${ }^{3}$ Department of Surgery, \\ Korea University Guro Hospital, Korea University College of Medicine, Seoul 152-703; \\ ${ }^{4}$ University of Ulsan College of Medicine, Asan Institute for Life Science, \\ Seoul 138-736, Republic of Korea
}

Received February 28, 2016; Accepted April 9, 2016

DOI: $10.3892 /$ or.2016.5086

\begin{abstract}
Recent studies have determined that inactivation of runt-related transcription factor 3 (RUNX3) expression is highly associated with lymph node metastasis and poor prognosis in various types of cancer. However, the mechanism of RUNX3-mediated suppression of tumor metastasis remains unclear. Herein, we aimed to clarify the effect of RUNX3 on metastasis and angiogenesis in colorectal cancer (CRC). Firstly, we found that the reduction in expression of RUNX3 in CRC tissues when compared with tumor adjacent normal colon tissues, as indicated by reduced RUNX3 staining, was significantly correlated with tumor-node-metastasis (TNM) stage. Secondly, we demonstrated that RUNX3 overexpression inhibited CRC cell migration and invasion resulting from the upregulation of matrix metalloproteinase-2 (MMP-2) and MMP-9 expression. In contrast, the knockdown of RUNX3 reduced the inhibition of migration and invasion of CRC cells. Finally, we found that restoration of RUNX3 decreased vascular endothelial growth factor (VEGF) secretion and suppressed endothelial cell growth and tube formation in CRC cells. All in all, our findings may provide insight into the
\end{abstract}

Correspondence to: Dr Byung Wook Min, Department of Surgery, Korea University Guro Hospital, Korea University College of Medicine, 148 Gurodong-ro, Guro-gu, Seoul 152-703, Republic of Korea

E-mail: gsmin@korea.ac.kr

Dr Dae-Hee Lee, Graduate School of Medicine, Korea University College of Medicine, 148 Gurodong-ro, Guro-gu, Seoul 152-703, Republic of Korea

E-mail: neogene@korea.ac.kr

*Contributed equally

Key words: colorectal cancer, runt-related transcription factor 3, migration, angiogenesis, VEGF development of RUNX3 for CRC metastasis diagnostics and therapeutics.

\section{Introduction}

Colorectal cancer (CRC) is one of the most common cancers worldwide. Despite recent advances in cancer therapy including chemotherapy, radiation and surgery, metastasis to other organs such as the lymph nodes, the liver and the lungs remains on ongoing issue (1). The survival rate of CRC patients has increased, but only by a relatively short period (20 months) when compared to other types of cancer $(2,3)$. A better understanding of the mechanisms involved in the pathways of metastasis may improve the management of CRC.

The runt-related transcription factor (RUNX) family consists of RUNX1, RUNX2 and RUNX3, which play an important role in cell proliferation and tumorigenesis (4). Among them, RUNX3, in particular, has been shown to play a tumor-suppressor role in several types of cancers and its expression levels are downregulated in tumor tissues $(5,6)$. RUNX3 was first suggested to be a tumor suppressor in gastric cancer (7). Recent studies have shown that RUNX3 expression levels are downregulated in CRC, either from hemizygous deletion or from promoter methylation of the RUNX3 gene. Furthermore, a decrease in RUNX3 protein expression was found to be significantly associated with decreased survival of CRC patients $(8,9)$. Mu et al $(10)$ showed that RUNX3 expression was decreased in $70 \%$ of primary human colorectal tumors; with over $90 \%$ of late stage tumors that represent highly metastatic tumors, showing reduced RUNX3 levels.

Epithelial-mesenchymal transition (EMT) is a morphogenetic process of the malignant transformation of epithelial cells. The EMT phenotype is characterized by the loss of cell-to-cell adhesion and remodeling of the actin cytoskeleton as well as the loss of the epithelial molecule E-cadherin and the gain of mesenchymal markers such as vimentin and Snail $(11,12)$. EMT is known to be necessary for cells to obtain migratory and invasive capabilities (13). It is necessary for tissue invasion, 
which is an essential step in metastasis that requires breakdown of the extracellular matrix (ECM) around the cancer cells. In the complicated multi-steps of cancer metastasis, one of the first steps is the degradation of the ECM by matrix metalloproteinases (MMPs). MMPs are mainly secreted during tumor development, invasion and metastasis; the secretion of MMPs promotes the invasion of malignant cancer cells into adjoining tissue, blood and lymph vessels. MMPs, particularly MMP-2 and MMP-9, have been postulated to promote invasion and lymph node metastasis of gastrointestinal cancer cells. Suppression of MMP activity impairs cancer cell migration and angiogenesis $(14,15)$, the recruitment of new blood vessels, which is required for invasiveness and metastasis $(16,17)$, and is regulated by the angiogenic stimulator vascular endothelial growth factor (VEGF) (18). Therefore, tumor angiogenesis and metastasis are key targets for anticancer therapy. It has been found that RUNX3 suppresses cell motility and metastasis in renal cell carcinoma and prostate cancer $(19,20)$. This suggests that RUNX3 plays an important role in tumorigenesis and progression. However, the role of RUNX3 in CRC has not yet been well studied.

In the present study, we found that deficiency or loss of RUNX3 expression was directly correlated with CRC tumor-node-metastasis (TNM) stage. Furthermore, restoration of RUNX3 expression led to the suppression of MMP-2 and MMP-9, and at least in part, to the inhibition of tumor metastasis. We also demonstrated that the reduction in VEGF release induced by RUNX3 reintroduction, inhibited CRC angiogenesis. Our clinical and mechanistic data indicated that RUNX3 may be a tumor suppressor involved in the progression of CRC.

\section{Materials and methods}

Reagents. RPMI-1640 medium, fetal bovine serum (FBS) and antibiotics were purchased from Gibco (Carlsbad, CA, USA). Antibodies obtained included RUNX3 (Abcam, Cambridge, MA, USA), E-cadherin (BD Biosciences, San Jose, CA, USA), $\beta$-actin (Sigma, St. Louis, MO, USA), vimentin (Dako, Carpinteria, CA, USA) and Snail (Santa Cruz Biotechnology, Santa Cruz, CA, USA). MMP-2 inhibitor was also purchased from Santa Cruz Biotechnology.

Cell lines and transfection. The human CRC cell lines (HCT116, DLD-1, SW480, SW620 and Colo205) were purchased from the Korea Cell Line Bank (KCLB) and maintained according to KCLB's instructions. Transfection of $1 \mu \mathrm{g}$ of plasmid DNA [pFlag-c1 (con) and pFlag-c1 RUNX3] into CRC cells was performed using Lipofectamine 2000 transfection reagent (Invitrogen, Carlsbad, CA, USA). Transfection of the RUNX3 siRNA and control siRNA (200 nM) was carried out using Lipofectamine RNAiMAX transfection reagent (Invitrogen). RUNX3 siRNA forward was 5'-GUG AUG GCA GGC AAU GAC GAG AAC U-3' and reverse, 5'-AGU UCU CGU CAU UGC CUG CCA UCA C-3'.

Wound-healing and Matrigel invasion assays. After transfection, the cells were seeded at $5 \times 10^{5}$ cells/well in 12 -well plates. At $100 \%$ confluence, two parallel wounds were made using a plastic pipette tip. The cells were then grown in culture medium with $5 \%$ FBS. Images of the wound were collected at 0,24 and $48 \mathrm{~h}$ using a microscope. The migration rate was quantified by measuring the distance between the wound edges. This assay was independently repeated three times. For the Matrigel invasion assay, $3 \times 10^{5}$ cells/well were seeded in the upper chamber, which was coated with Matrigel (BD Biosciences). After $48 \mathrm{~h}$ at $37^{\circ} \mathrm{C}$ in $5 \% \mathrm{CO}_{2}$, the cells present on the lower surface of the insert were stained with Diff-Quik Stain kit (Biochemical Sciences, Inc., Swedesboro, NJ, USA). The cells invaded through the Matrigel-coated membrane were counted by microscopy.

Cell viability assay. CRC cells were grown in a 96-well plate at $1 \times 10^{4}$ cells/well. The cells were transfected with pFlag-cl RUNX3 or pFlag-cl (con). At $24 \mathrm{~h}$ after transfection, $25 \mu \mathrm{l}$ of 3-(4,5-dimethylthiazol-2-yl)-2,5-diphenyltetrazolium bromide (MTT) $(5 \mathrm{mg} / \mathrm{ml})$ (Sigma-Aldrich Co. LLC, St. Louis, MO, USA) was added to each well, and the plates were incubated for $4 \mathrm{~h}$ at $37^{\circ} \mathrm{C}$. The MTT solution in the medium was then aspirated, and $150 \mu \mathrm{l}$ of dimethyl sulfoxide (DMSO) was added before measurement of the absorbance at $550 \mathrm{~nm}$.

Colony formation assay. Stably transfected HCT116 and DLD-1 cells were diluted and seeded into 6-well plates at a density of 500 cells/well, and then were cultured at $37^{\circ} \mathrm{C}$. The medium was changed every three days. After one week, the cells were washed with phosphate-buffered saline (PBS), fixed with $4 \%$ paraformaldehyde for $30 \mathrm{~min}$, and then stained with crystal violet for $30 \mathrm{~min}$ for visualization and counting.

Immunofluorescence. Cells grown on a glass coverslip were fixed with $3.7 \%$ formaldehyde for $15 \mathrm{~min}$, followed by permeabilization with $0.5 \%$ Triton X-100 for $15 \mathrm{~min}$ at room temperature. The cells were then blocked for $1 \mathrm{~h}$ with $3 \%$ bovine serum albumin. Primary antibodies were applied overnight at $4{ }^{\circ} \mathrm{C}$, followed by incubation with secondary Alexa Fluor 594-conjugated secondary antibody (Molecular Probes, Eugene, OR, USA) or the FITC-conjugated secondary antibody (Sigma). The nuclei were costained with 4',6-diamidino-2-phenylindole (DAPI) and visualized using fluorescence microscopy.

Western blotting. Cells were lysed in RIPA buffer [50 mM Tris, $150 \mathrm{mM} \mathrm{NaCl}, 1 \%$ Triton $\mathrm{X}-100,0.1 \% \mathrm{SDS}$ and $1 \%$ Na-deoxycholate ( $\mathrm{pH} 7.4)]$ with proteases and phosphatase inhibitor cocktails and were subjected to SDS-PAGE. Cells were then transferred onto nitrocellulose membranes (GE Healthcare Life Sciences, Logan, UT, USA), blocked with TBS containing $0.2 \%$ Tween-20 and 5\% skim milk, incubated with the primary antibody and then incubated with the horseradish peroxidase-labeled secondary antibody. The signals were detected by X-ray film.

RT-PCR and real-time PCR. Total RNA was extracted using TRIzol reagent (Life Technologies, Grand Island, NY, USA). Amplification of transcripts was performed by reverse transcriptase polymerase chain reaction kit (Life Technologies). RT-PCR primers for MMP-2 were: forward, 5'-TGA TGG TGT CTG CTG GAA AG-3' and reverse, 5'-GAC ACG TGA AAA GTG CCT TG-3'); and for MMP-9 forward, 5'-AAG 

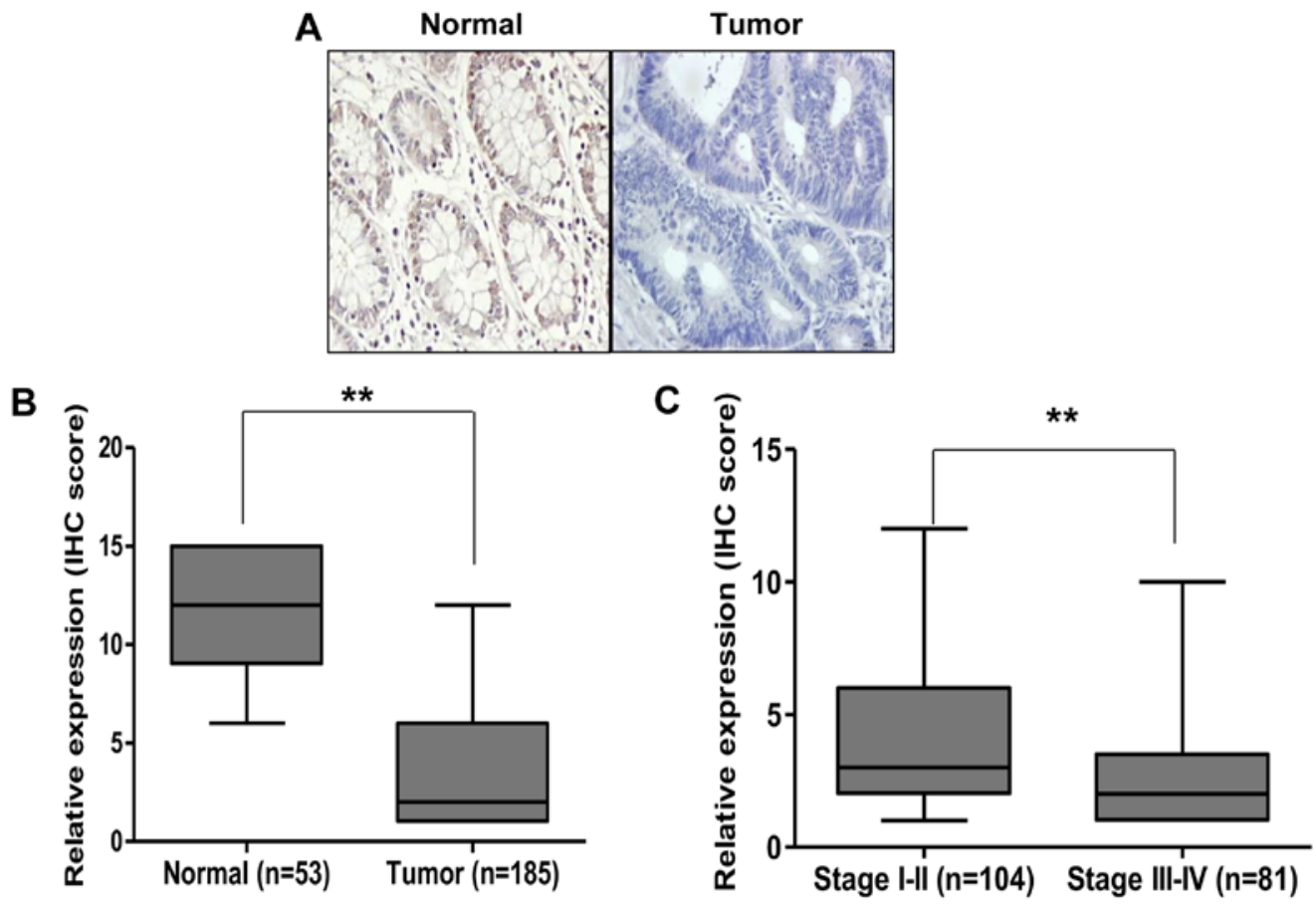

Figure 1. RUNX3 expression in tumor adjacent normal colorectal and CRC tissues. (A) Representative images of immunohistochemistry in human CRC specimens stained for RUNX3. Magnification, x40. (B) Box plots indicate the percentage of RUNX3-positive cells in normal (left box) and tumor samples (right box); ${ }^{* *} \mathrm{P}<0.01$ for normal vs. tumor tissues. Normal, $\mathrm{n}=53$; tumor, $\mathrm{n}=185$. The data for the box plots are derived from the immunohistochemical analyses in A. (C) Expression of RUNX3 is correlated with TNM stage. ${ }^{* *} \mathrm{P}<0.01$, when comparing I-II vs. III-IV stage tissues.

ATG CTG CTG TTC AGC GGG-3' and reverse, 5'-GTC CTC AGG GCA CTG CAG GAT-3'). Real-time PCR was performed on an Applied Biosystems 9700 real-time PCR using gene specific oligonucleotide primers for TaqMan probes (Applied Biosystems, Foster City, CA, USA). TaqMan probes were as follows: GAPDH (Hs99999905_m1), RUNX3 (Hs00231709_ m1) and MMP-2 (Hs01548727_m1). For expression of mRNA, gene expression was normalized to GAPDH.

Tube formation assay. For the tube formation assay, a 48-well plate was coated with Matrigel (BD Biosciences). Human umbilical vein endothelial cells (HUVECs) $\left(3 \times 10^{4}\right)$ were suspended in $100 \mu \mathrm{l}$ Medium 200PRF (Life Technologies) and applied to the pre-coated 48-well plate. After $6 \mathrm{~h}$, the number of capillary-like tubes was counted using microscopy.

Enzyme-linked immunosorbent assay (ELISA) for VEGF. HCT116 and DLD-1 cells were seeded in a 60-mm dish. The supernatants were collected at $48 \mathrm{~h}$. The levels of VEGF secreted by the cells in the medium were detected using VEGF ELISA kit (R\&D Systems, Minneapolis, MN, USA).

\section{Results}

RUNX3 expression is decreased in human CRC. We first investigated whether expression of RUNX3 is reduced in human CRC. Immunohistochemistry (IHC) staining was performed on TMA slides containing normal and tumor cancer tissues. As shown in Fig. 1A, from the serial sections of paired normal colorectal and tumor tissues of CRC patients, RUNX3 was highly expressed in the normal colorectal tissues, but lower in the tumor tissues. Our IHC data showed that there was a significantly lower level of RUNX3 in the tumor tissues than that noted in the normal colon tissues $(\mathrm{P}<0.01$, Fig. 1B). Since TNM stage is an important prognostic and metastatic marker for patients, we confirmed expression of RUNX3 in stage I-II $(n=104)$ and stage III-IV $(n=81)$ CRC tissues. We found that expression of RUNX3 was markedly decreased in stage III-IV compared with stage I-II tissues (Fig. 1C). These results suggest that RUNX3 may be an important prognostic marker in CRC patients.

RUNX3 does not affect cell viability and proliferation in CRC cells. A previous study reported that loss of RUNX3 induces proliferative effect in gastric cancer cells (21). To investigate the function of RUNX3 in CRC, we tested whether RUNX3 affects the viability of CRC cells. We first determined the endogenous protein level of RUNX3 in CRC cell lines. As shown in Fig. 2A, HCT116, SW480 and SW620 cells expressed a higher RUNX3 level than corresponding DLD-1 and Colo205 cells. Then, after pFlag-c1 RUNX3 or pFlag-c1 (con) transfection, the cell viability of the CRC cells was performed by MTT assay, but there was no change in the RUNX3overexpressing cells (Fig. 2B). In order to further confirm cell proliferation, we performed a colony formation assay. As shown in Fig. 2C and D, there was no significant difference in colony formation ability in RUNX3-overexpressing HCT116 and DLD-1 cells compared with this ability in cells transfected with pFlag-cl (control).

RUNX3 plays an important role in motility and invasiveness. Since Fig. 1A demonstrates that expression of RUNX3 is associated with TNM stage, RUNX3 may play an important role in CRC metastasis. Thus, we performed an experiment 

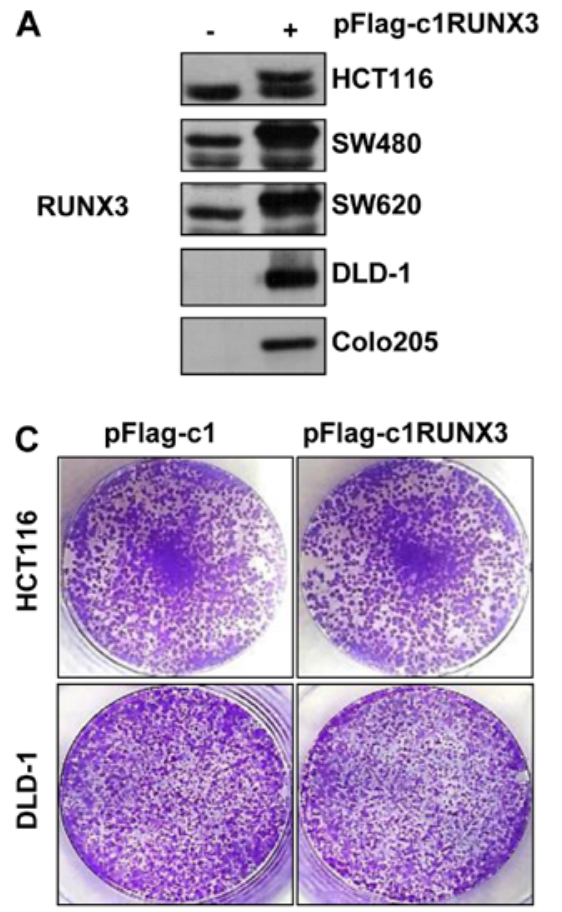

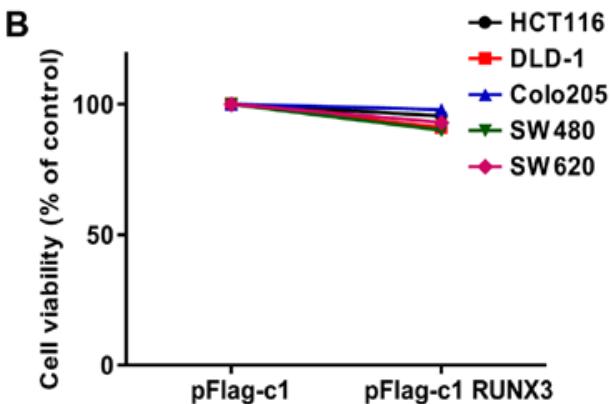

D

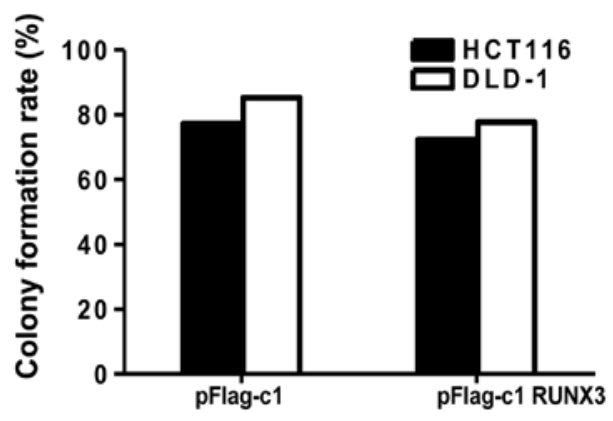

Figure 2. RUNX3 does not affect cell viability and proliferation in CRC cells. (A) The endogenous protein level of RUNX3 in CRC cell lines was assessed by western blotting. (B) The cell viability of the CRC cells was determined by MTT assay after transfection with pFlag-c1 RUNX3 or pFlag-c1 (con) for $24 \mathrm{~h}$. (C and D) The effect of cell growth with or without RUNX3 on colony formation of HCT116 and DLD-1 cells. (C) Representative images of the colonyformation assay. (D) Analysis of colony formation rates in the HCT116 and DLD-1 cells with or without RUNX3. Data are presented as the means \pm SD of three independent experiments.

to test whether overexpression of RUNX3 could prevent EMT. First, we examined EMT markers at the protein levels in the RUNX3-overexpressing cells. As shown in Fig. 3A, epithelial marker E-cadherin was significantly increased while mesenchymal markers vimentin and Snail were decreased in the RUNX3-overexpressing cells compared with these levels in pFlag-cl (con) cells. These observations were also confirmed by immunofluorescence. E-cadherin expression in the RUNX3-overexpressing cells was increased and vimentin expression in the RUNX3-overexpressing cells was decreased (Fig. 3B). We next investigated the effect of RUNX3 on the cell motility of the CRC cells. Cell motility was significantly decreased in the RUNX3-overexpressing cells. In contrast, cell migration was also increased in the RUNX3 siRNA-transfected HCT116 and DLD-1 cells (Fig. 3C). In addition, to examine the effect of RUNX3 expression on the metastatic ability of CRC cells, HCT116 and DLD-1 cells were transiently transfected before performing Matrigel invasion assay. The RUNX3-overexpressing cells had decreased cell invasive ability while knockdown of RUNX3 increased the cell invasive ability compared with that of the cells transfected with control siRNA (Fig. 3D). The results suggest that RUNX3 acts as a key regulator of metastasis and EMT.

RUNX3 is a regulator of MMPs for invasiveness and angiogenesis. To study the role of RUNX3 in cell invasion, we measured the activities of MMP-2 and MMP-9 by RT-PCR . As shown in Fig. 4A, the activity of MMP-2 was increased, but not that of MMP-9, in the RUNX3 siRNA-transfected HCT116 and DLD-1 cells compared with the activities in the con siRNA cells. Hence, we hypothesized that MMP-2 is affected by the invasive effects of cells transfected with RUNX3 siRNA. To test this hypothesis, we treated the cells with the MMP-2 inhibitor $(5 \mu \mathrm{M})$ for $24 \mathrm{~h}$ after transfection of RUNX3 siRNA. Cell motility promoted by RUNX3 siRNA was significantly decreased by the MMP-2 inhibitor (Fig. 4B). We then assessed the effects of the MMP-2 inhibitor on the invasion of RUNX3 siRNA-transfected HCT116 and DLD-1 cells. As shown in Fig. 4C, the cells treated with the MMP-2 inhibitor showed significantly lower invasiveness. Together, these data demonstrated that RUNX3 inhibits metastasis through MMP-2. To further examine the effect of RUNX3 on angiogenesis, we performed an endothelial cell tube formation assay. The degree of tube formation was assessed as the percentage of cell surface area vs. total surface area. As shown in Fig. 4D, the average number of complete tubular structures formed by HUVECs was significantly reduced in the conditioned medium from the RUNX3-overexpressing HCT116 and DLD-1 cells compared with the vector controls. To evaluate the mechanism involved in the regulation of angiogenesis by RUNX3, ELISA was performed to detect VEGF secretion into the conditioned culture medium of CRC cells. As shown in Fig. 4E, a significant reduction in VEGF secretion was observed in the conditioned medium from the HCT116 and DLD-1 cells transfected with pFlag-RUNX3 compared with the control cells $(\mathrm{P}<0.05)$.

\section{Discussion}

In previous studies, RUNX3 in colorectal cancer (CRC) has been considered to be associated with cell apoptosis and hypermethylation $(22,23)$. However, the effect of RUNX3 
A

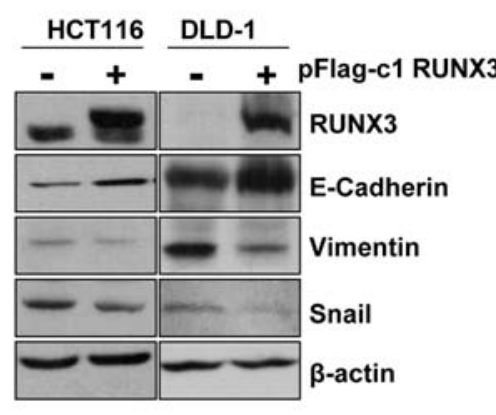

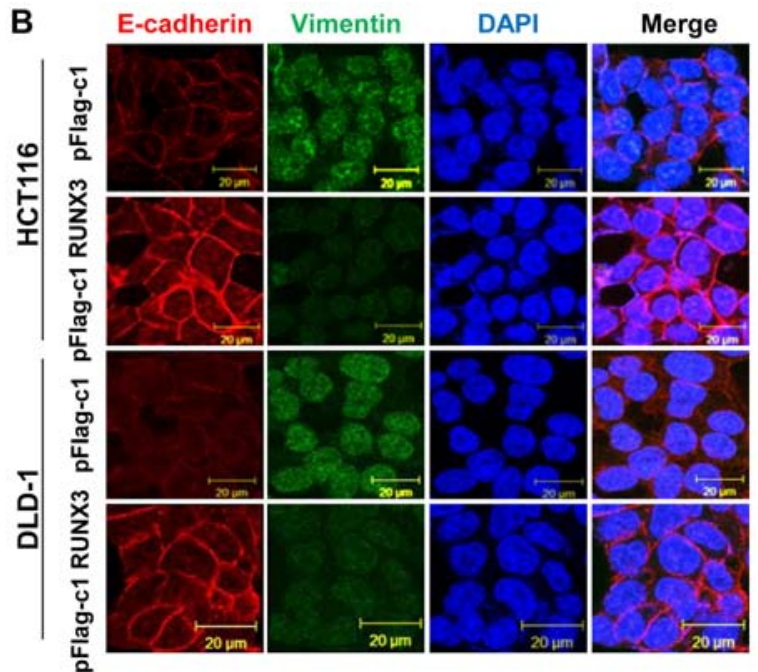
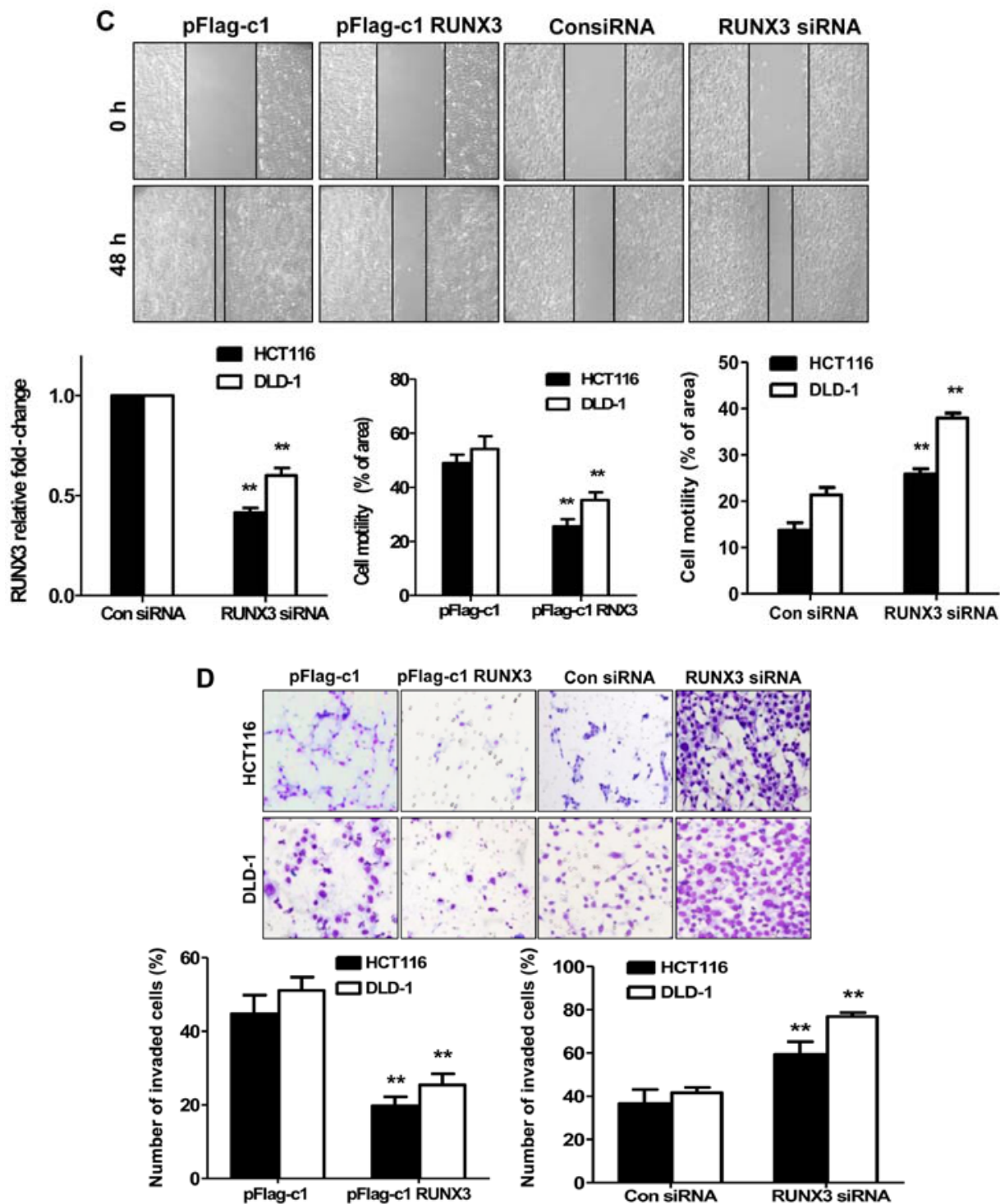

Figure 3. Effect of RUNX3 on the invasion and metastatic abilities of CRC, HCT116 and DLD-1 cells. (A) Western blotting was performed to detect the levels of EMT markers (E-cadherin, vimentin and Snail) and RUNX3. (B) The expression levels of E-cadherin and vimentin were detected by immunofluorescence. The nuclei were counterstained with DAPI. E-cadherin (red) and vimentin (green) were visualized by confocal fluorescence microscopy (original magnification, $\mathrm{x} 40$ ). Scale bar, $20 \mu \mathrm{M}$. (C) Wound-healing assay was performed to determine the effect of RUNX3 on cell motility. Cell monolayers were scratched with a pipette tip and incubated with 5\% FBS medium. Cell migration to the wound area was then monitored for $48 \mathrm{~h}$ post-wound, and the percentage of total area covered by the cells was then assessed using the NIH Image program. P-values were calculated compared with pFlag-c1 (con), ${ }^{* *} \mathrm{P}<0.01$. (D) Transwell assays with Matrigel were performed to detect the effect of RUNX3 on the invasive ability of CRC, HCT116 and DLD-1 cells. The invaded cells on the bottom chamber were stained with crystal violet and counted. Images of invasive HCT116 and DLD-1 cells (top panel). Quantitative analyses for the cell invasion through the Matrigel-coated membrane (bottom panel); ${ }^{* *} \mathrm{P}<0.01$. 
A

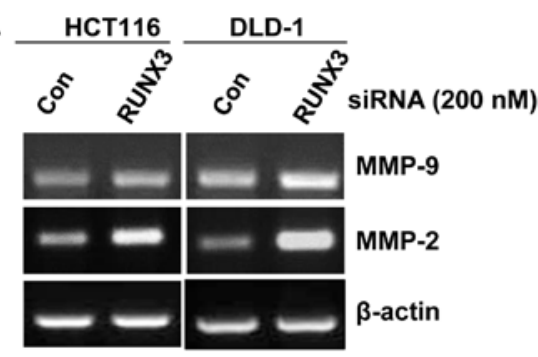

B
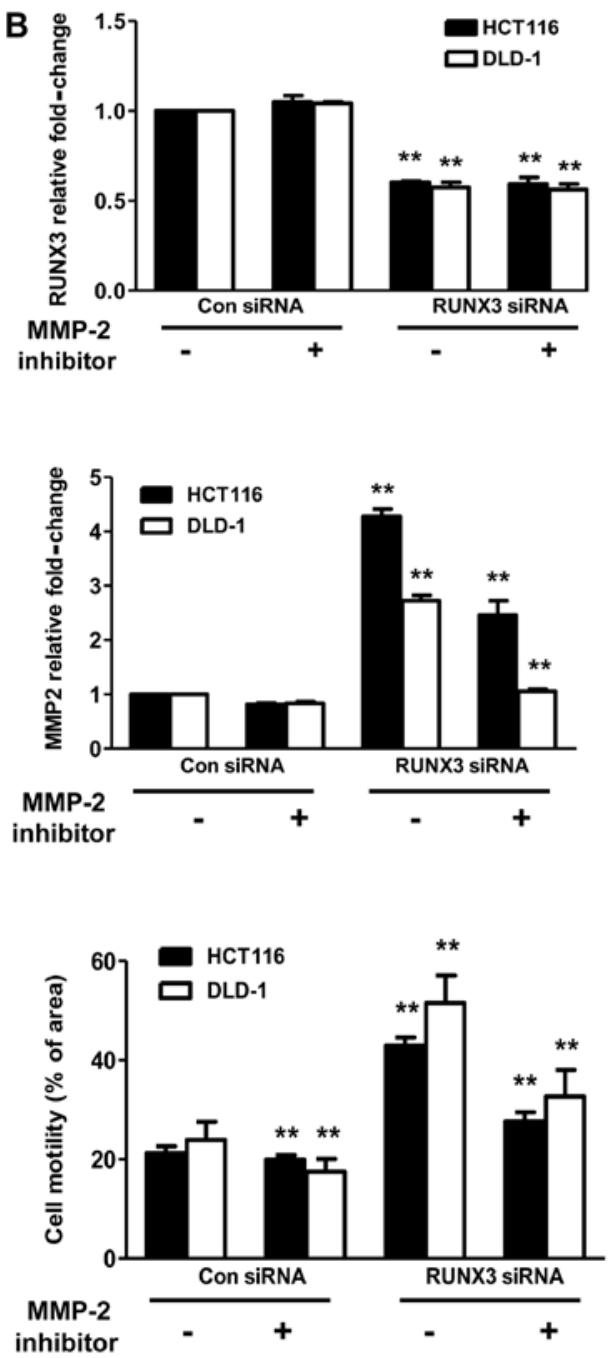
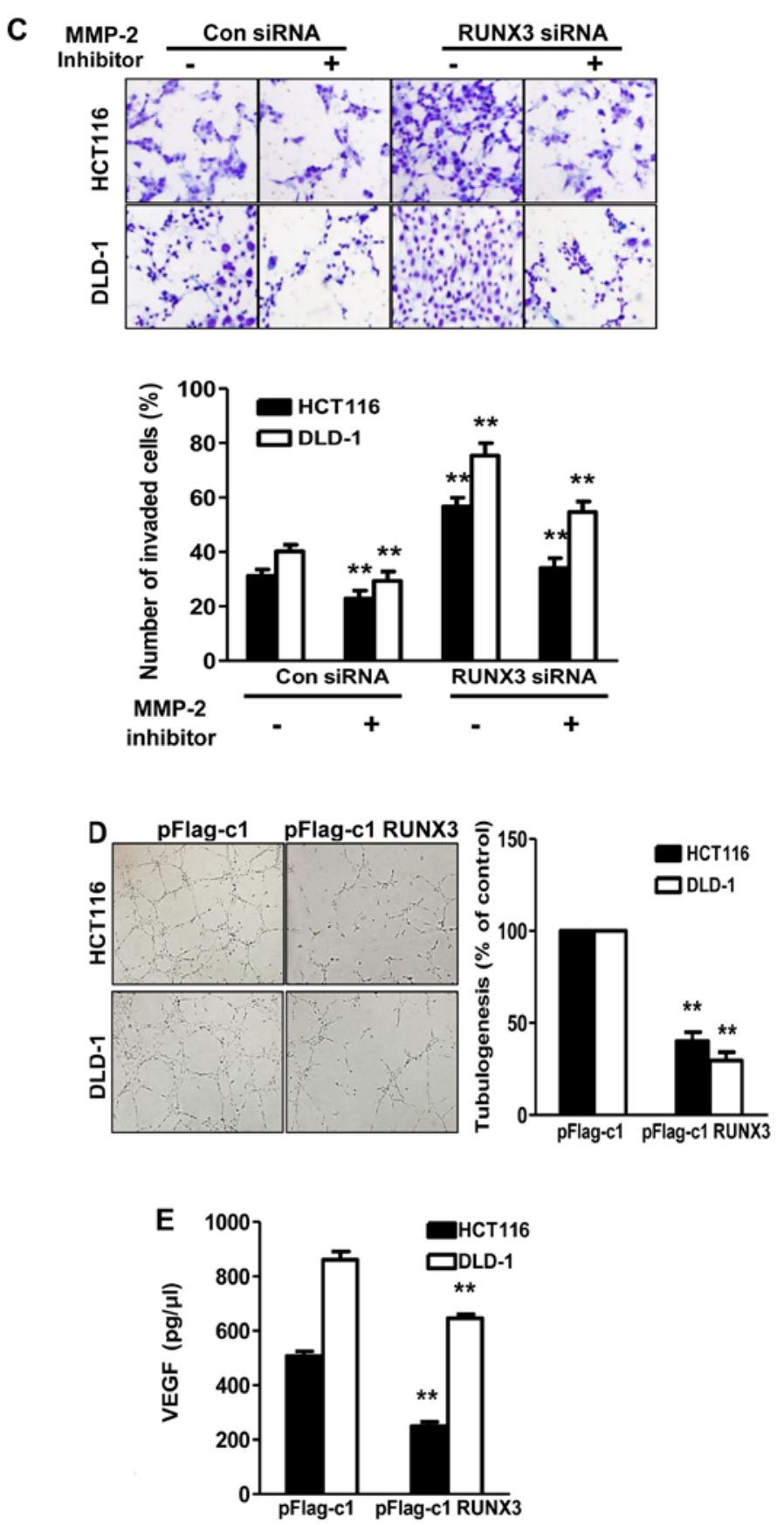

Figure 4. RUNX3 inhibits angiogenesis by suppression of MMPs. (A) HCT116 and DLD-1 cells were transfected with RUNX3 siRNA or non-specific (con) siRNA. In addition, the expression levels of MMP-2 and MMP-9 were analyzed by RT-PCR. $\beta$-actin served as a control for total RNA. (B) HCT116 and DLD-1 cells transfected with RUNX3 siRNA or non-specific (con) siRNA were treated with an MMP-2 inhibitor (5 $\mu \mathrm{M})$ for $24 \mathrm{~h}$. RUNX3 and MMP-2 mRNA levels were determined by real-time PCR. Relative fold-change levels were normalized to the corresponding GAPDH mRNA expression level. Wound-healing assay (bottom left graph) was performed to determine the effect of MMP-2 on cell motility. Cell monolayers were scratched with a pipette tip and incubated with 5\% FBS medium. Cell migration to the wound area was then monitored for $48 \mathrm{~h}$ post-wound, and the percentage of total area covered by cells was then assessed using the NIH Image program. P-values were calculated compared with pFlag-cl (con); ${ }^{* *} \mathrm{P}<0.01$. (C) Transwell assays with Matrigel were performed to detect the invasive ability of the MMP-2 inhibitor. The invaded cells on the bottom were stained with crystal violet and counted. Images of invasive HCT116 and DLD-1 cells are shown (upper panel). Quantitative analyses of the cell invasion through the Matrigel-coated membrane (lower panel) ${ }^{*} \mathrm{P}<0.05,{ }^{* *} \mathrm{P}<0.01$. (D) The angiogenic potential of colon cancer cells was determined by an endothelial cell tube formation assay. Conditioned media (CM) was prepared from HCT116 and DLD-1 cells transiently transfected with pFlag-c1 (con) or pFlag-c1 RUNX3. HUVECS $\left(5 \times 10^{4}\right)$ in $200 \mu 1$ of CM were then plated on growth factor-reduced Matrigel to form capillary tubes. Capillary tube formation in each group was captured using a light microscope. All experiments were carried out in triplicate. (E) Quantification of VEGF secretion in HCT116 and DLD-1 cells transduced with pFlag-c1 (con) and pFlag-c1 RUNX3 using an ELISA kit. $\mathrm{P}$-values were calculated compared with pFlag-c1 (con); ${ }^{* *} \mathrm{P}<0.01$.

alteration on tumor angiogenesis and metastasis has not been examined in CRC. In the present study, we found that RUNX3 was frequently silenced or downregulated in CRC cell lines, as well as in primary CRC compared to normal colorectal mucosa tissues. Specifically, we found that the expression of RUNX3 was decreased in the late stage III-IV compared to 
the early stage I-II tumor samples (Fig. 1C). These clinical data clearly demonstrated that expression of RUNX3 affects cancer metastasis in CRC. Finally, functional experiments revealed that restoration of RUNX3 in CRC cells inhibited cell proliferation, suppressed cell invasion and migration and regulated EMT-related proteins, E-cadherin, vimentin and Snail. All things considered, our data suggest that RUNX3 is a tumor-suppressor gene, which is downregulated through promoter hypermethylation in CRC.

The major cause of mortality of cancer patients is metastasis (24). The EMT process in tumor cells is an essential step in metastasis. Thus, we detected changes in expression of epithelial marker E-cadherin and mesenchymal markers Snail and vimentin corresponding to changes in RUNX3 expression. In the present study, knockdown of RUNX3 expression markedly weakened the migration and invasion ability of the HCT116 and DLD-1 cells compared with the negative control (Fig. 3). It has also been found that downregulation of the epithelial marker E-cadherin induced the expression of various mesenchymal markers, such as Snail and vimentin, during EMT (25). Consistent with these observations, we found that overexpression of RUNX3 significantly inhibited motility and invasiveness in CRC cells. Whereas, knockdown of RUNX3 promoted motility and invasiveness in the HCT116 and DLD-1 cells (Fig. 3). These results indicated that RUNX3 may inhibit EMT progression in CRC cells.

It is possible that MMPs degrade components of the basement membrane and ECM, allowing cancer cells to invade and migrate $(26,27)$. In addition, several studies have demonstrated that MMP-2 and MMP-9 promote invasion and metastasis in CRC (28). To determine how RUNX3 inhibits CRC cell migration and invasion, we focused on clarifying the relationship between RUNX3 and MMPs, which have been reported to participate in tumor progression. In the present study, we found that RUNX3 overexpression significantly inhibited the expression of MMP-2 and MMP-9 in HCT116 and DLD-1 cells (Fig. 4A-C). We found that knockdown of RUNX3 induced expression of MMP-2 but not of MMP-9. Our data imply that RUNX3 may suppress CRC cell invasion and migration by decreasing MMP-2 and MMP-9 protein expression. However, it remains to be elucidated how RUNX3 regulates MMP-2 and MMP-9 expression and how the signaling pathway of RUNX3 regulates $\mathrm{CRC}$ cell invasion.

VEGF, an angiogenic factor, has been identified as a key mediator of tumor angiogenesis involved in the development of tumor blood supply in the progression of solid tumors (29). Previous studies have demonstrated that RUNX3 downregulated VEGF expression via transcriptional repression in human gastric cancer and renal cancer cells $(19,30)$. We detected the expression and secretion of VEGF after RUNX3 transfection. Our data showed that VEGF secretion was reduced by restoration of RUNX3 (Fig. 4D and E). These results suggested that RUNX3 suppresses blood vessel formation by regulating VEGF secretion.

In summary, the present study revealed that the tumor-suppressor gene RUNX3 was inactivated by promoter methylation in CRC cell lines and primary CRC tissues. Restoration of RUNX3 enhanced cell invasion and migration, but had no effect on cell proliferation. Consequently, these findings show that the knockdown or restoration of RUNX3 presents a possible means for inhibiting angiogenesis or metastasis and for inhibiting CRC progression.

\section{Acknowledgements}

The present study was supported by the Basic Science Research Program through the National Research Foundation of Korea (NRF) funded by the Ministry of Education, Science and Technology (no. NRF-2013R1A1A2064367) and the Hanmi Pharm. Co., Ltd. (no. 11500841).

\section{References}

1. Geoghegan JG and Scheele J: Treatment of colorectal liver metastases. Br J Surg 86: 158-169, 1999.

2. Yokota J: Tumor progression and metastasis. Carcinogenesis 21: 497-503, 2000.

3. Fidler IJ: The pathogenesis of cancer metastasis: The 'seed and soil' hypothesis revisited. Nat Rev Cancer 3: 453-458, 2003.

4. Lund AH and van Lohuizen M: RUNX: A trilogy of cancer genes. Cancer Cell 1: 213-215, 2002.

5. Bae SC and Choi JK: Tumor suppressor activity of RUNX3. Oncogene 23: 4336-4340, 2004.

6. Subramaniam MM, Chan JY, Yeoh KG, Quek T, Ito K and Salto-Tellez M: Molecular pathology of RUNX3 in human carcinogenesis. Biochim Biophys Acta 1796: 315-331, 2009.

7. LiQL, Ito K, Sakakura C, Fukamachi H, Inoue K, Chi XZ, Lee KY, Nomura S, Lee CW, Han SB, et al: Causal relationship between the loss of RUNX3 expression and gastric cancer. Cell 109: 113-124, 2002.

8. Soong R, Shah N, Peh BK, Chong PY, Ng SS, Zeps N, Joseph D, Salto-Tellez M, Iacopetta B and Ito Y: The expression of RUNX3 in colorectal cancer is associated with disease stage and patient outcome. Br J Cancer 100: 676-679, 2009.

9. Silva TD, Vidigal VM, Felipe AV, DE Lima JM, Neto RA, Saad SS and Forones NM: DNA methylation as an epigenetic biomarker in colorectal cancer. Oncol Lett 6: 1687-1692, 2013.

10. Mu WP, Wang J, Niu Q, Shi N and Lian HF: Clinical significance and association of $R U N X 3$ hypermethylation frequency with colorectal cancer: A meta-analysis. Onco Targets Ther 7: $1237-1245,2014$

11. Thiery JP: Epithelial-mesenchymal transitions in development and pathologies. Curr Opin Cell Biol 15: 740-746, 2003.

12. Bedi S, Vidyasagar A and Djamali A: Epithelial-to-mesenchymal transition and chronic allograft tubulointerstitial fibrosis. Transplant Rev 22: 1-5, 2008.

13. Tsai JH and Yang J: Epithelial-mesenchymal plasticity in carcinoma metastasis. Genes Dev 27: 2192-2206, 2013.

14. Wagenaar-Miller RA, Gorden L and Matrisian LM: Matrix metalloproteinases in colorectal cancer: Is it worth talking about? Cancer Metastasis Rev 23: 119-135, 2004.

15. Zucker S and Vacirca J: Role of matrix metalloproteinases (MMPs) in colorectal cancer. Cancer Metastasis Rev 23: 101-117, 2004.

16. Zetter BR: Angiogenesis and tumor metastasis. Annu Rev Med 49: 407-424, 1998 .

17. Folkman J: Role of angiogenesis in tumor growth and metastasis. Semin Oncol 29 (Suppl 16): S15-S18, 2002.

18. Leung DW, Cachianes G, Kuang WJ, Goeddel DV and Ferrara N: Vascular endothelial growth factor is a secreted angiogenic mitogen. Science 246: 1306-1309, 1989.

19. Chen F, Bai J, Li W, Mei P, Liu H, Li L, Pan Z, Wu Y and Zheng J: RUNX3 suppresses migration, invasion and angiogenesis of human renal cell carcinoma. PLoS One 8: e56241, 2013.

20. Chen F, Wang M, Bai J, Liu Q, Xi Y, Li W and Zheng J: Role of RUNX3 in suppressing metastasis and angiogenesis of human prostate cancer. PLoS One 9: e86917, 2014.

21. Cheng HC, Liu YP, Shan YS, Huang CY, Lin FC, Lin LC, Lee L, Tsai CH, Hsiao M and Lu PJ: Loss of RUNX3 increases osteopontin expression and promotes cell migration in gastric cancer. Carcinogenesis 34: 2452-2459, 2013.

22. Tong DD, Jiang Y, Li M, Kong D, Meng XN, Zhao YZ, Jin Y, Bai J, Fu SB and Geng JS: RUNX3 inhibits cell proliferation and induces apoptosis by TGF-beta-dependent and -independent mechanisms in human colon carcinoma cells. Pathobiology 76: 163-169, 2009. 
23. Nishio M, Sakakura C, Nagata T, Komiyama S, Miyashita A, Hamada T, Kuryu Y, Ikoma H, Kubota T, Kimura A, et al: RUNX3 promoter methylation in colorectal cancer: Its relationship with microsatellite instability and its suitability as a novel serum tumor marker. Anticancer Res 30: 2673-2682, 2010.

24. Tam WL and Weinberg RA: The epigenetics of epithelial-mesenchymal plasticity in cancer. Nat Med 19: 1438-1449, 2013.

25. Wang M, Zhao F, Li S, Chang AK, Jia Z, Chen Y, Xu F, Pan H and Wu H: AIB1 cooperates with ER $\alpha$ to promote epithelial mesenchymal transition in breast cancer through SNAI1 activation. PLoS One 8: e65556, 2013.

26. Rundhaug JE: Matrix metalloproteinases and angiogenesis. J Cell Mol Med 9: 267-285, 2005.

27. Deryugina EI and Quigley JP: Matrix metalloproteinases and tumor metastasis. Cancer Metastasis Rev 25: 9-34, 2006.
28. Yang B, Tang F, Zhang B, Zhao Y, Feng J and Rao Z: Matrix metalloproteinase- 9 overexpression is closely related to poor prognosis in patients with colon cancer. World J Surg Oncol 12: 24, 2014.

29. Cao Y, e G, Wang E, Pal K, Dutta SK, Bar-Sagi D and Mukhopadhyay D: VEGF exerts an angiogenesis-independent function in cancer cells to promote their malignant progression. Cancer Res 72: 3912-3918, 2012.

30. Peng Z, Wei D, Wang L, Tang H, Zhang J, Le X, Jia Z, Li Q and Xie K: RUNX3 inhibits the expression of vascular endothelial growth factor and reduces the angiogenesis, growth, and metastasis of human gastric cancer. Clin Cancer Res 12: 6386-6394, 2006. 